JOURNAL OF

SYMPLECTIC GEOMETRY

Volume 3, Number 3, 341-355, 2005

\title{
SYMPLECTIC DEFORMATIONS OF KÄHLER MANIFOLDS
}

\author{
PAOlo de BARTolomeis
}

\begin{abstract}
Given a compact symplectic manifold $(M, \kappa), H^{2}(M, \mathbb{R})$ represents, in a natural sense, the tangent space of the moduli space of germs of deformations of the symplectic structure. In the case $(M, \kappa, J)$ is a compact Kähler manifold, the author provides a complete description of the subset of $\boldsymbol{H}^{2}(\boldsymbol{M}, \mathbb{R})$ corresponding to Kähler deformations, including the non-generic case, where (at least locally) some hyperkähler manifold factors out from $\boldsymbol{M}$. Several examples are also discussed.
\end{abstract}

\section{Introduction}

The naif deformation theory of symplectic manifolds is quite simple: let $(M, \kappa)$ be a compact symplectic manifold and let $\alpha \in \wedge^{2}(M, \mathbb{R}), d \alpha=0$ : then

$$
\kappa_{t}:=\kappa+t \alpha
$$

is a (germ of) curve of symplectic structures having tangent $\alpha$ at 0 ; moreover, Moser's lemma (cf. [2]) ensures that $\kappa_{t}=\phi_{t}^{*}(\kappa)$ for a path of diffeomorphisms with $\phi_{0}=i d_{M} \Longleftrightarrow \alpha=d \beta$ and so $H^{2}(M, \mathbb{R})$ is the tangent space of the moduli space of germs of deformations of symplectic structures and the theory is totally unobstructed (for a non-naïf version, see $[\mathbf{1}])$.

Let $(M, \kappa, J)$ be a compact Kähler manifold: therefore, $J$ is a $\kappa$ calibrated holomorphic structure and so $g=g_{J}:=\kappa(J \cdot, \cdot)$ is a positive definite Hermitian metric; we want to investigate the subset of $H^{2}(M, \mathbb{R})$ corresponding to Kähler deformations of $\kappa$.

We have the following

Theorem 1.1. Let $(M, \kappa, J)$ be a compact Kähler manifold; let $\mathcal{K}$ be the subset of $H^{2}(M, \mathbb{R})$ corresponding to Kähler deformations of $\kappa$; i.e., $[\alpha] \in \mathcal{K}$ if and only if there exists a curve of Kähler structures $\left(\kappa_{t}, J_{t}\right)$ with $\kappa_{t}=$ $\kappa+t \alpha+o(t), J_{0}=J$ 
then:

$$
\mathcal{K}=\mathcal{P}^{2,0+0,2} \oplus H^{1,1}(M, \mathbb{R})
$$

where

$$
\mathcal{P}^{2,0+0,2}(M):=\left\{a \in H^{2,0+0,2}(M, \mathbb{R}) \mid \nabla^{M} h(a)=0\right\}
$$

and $h(a)$ is the $g$-harmonic representative of $a$.

Note that, clearly, $\mathcal{P}^{2,0+0,2}(M)$ is generically reduced to $\{0\}$ and, if it is not the case, then (at least locally) some hyperkähler manifold factors out from $M$.

The author is pleased to thank the referee for valuable remarks and suggestions for a better presentation of the results.

\section{Reduction to the $(2,0+0,2)$-case}

We have first the following

Lemma 2.1. Let $(M, \kappa, J)$ be a compact Kähler manifold;

then:

$$
\mathcal{K}+H^{1,1}(M, \mathbb{R})=\mathcal{K}
$$

i.e., for every $a \in \mathcal{K}$, every $c \in H^{1,1}(M, \mathbb{R})$, we have $a+c \in \mathcal{K}$.

Proof. Let $\alpha \in \wedge^{2}(M, \mathbb{R}), d \alpha=0$, such that $[\alpha] \in \mathcal{K}$.

Given $c \in H^{1,1}(M, \mathbb{R})$, let $\gamma \in \wedge_{J}^{1,1}(M)$ be its harmonic representative;

by assumption, there is a curve of Kähler structures $\left(\kappa_{t}, J_{t}\right)$ with $\kappa_{t}=\kappa+t \alpha+o(t)$; by Kodaira-Spencer theory, the projection

$$
P_{t}: \wedge_{J_{t}}^{1,1}(M) \longrightarrow \mathcal{H}_{g_{J_{t}}}^{1,1}(M)
$$

(where, of course, $\mathcal{H}_{g_{J_{t}}}^{1,1}(M)$ is the space of $g_{J_{t}}$-harmonic $(1,1)$-forms on $M$ ) is smooth in $t$ (see e.g., [3], p. 184).

Let

$$
\check{\kappa}_{t}:=\kappa_{t}+\frac{1}{2} t\left(\gamma+J_{t} \gamma\right)
$$

i.e.,

$$
\check{\kappa}(X, Y)=\kappa_{t}(X, Y)+\frac{1}{2} t\left(\gamma(X, Y)+\gamma\left(J_{t} X, J_{t} Y\right)\right)
$$

and

$$
\tilde{\kappa}_{t}:=P_{t}\left(\check{\kappa}_{t}\right)=\kappa_{t}+\frac{1}{2} t P_{t}\left(\gamma+J_{t} \gamma\right)
$$

Clearly $\left(\tilde{\kappa}_{t}, J_{t}\right)$ is a curve of Kähler structures (note: the same $J_{t}$ 's!) and

$$
\frac{d \tilde{\kappa}_{t}}{d t}{ }_{\mid t=0}=\alpha+\frac{1}{2} P_{0}(\gamma+J \gamma)=\alpha+\gamma
$$




\section{The main result}

Let us first recall the basic linear algebraic frame: let $(T, J, g)$ be a Hermitian vector space, i.e., a real vector space $T$ equipped with $J \in \operatorname{End}(T)$ satisfying $J^{2}=-I$ and a positive definite scalar product $g$ on $T$ satisfying $g(J X, J Y)=g(X, Y)$;

then

$$
T^{\mathbb{C}}=T^{1,0} \oplus T^{0,1}
$$

and

$$
\nu: T \longrightarrow T^{1,0}, \quad \nu(X):=\frac{1}{2}(X-i J X)
$$

is a linear isomorphism such that $\nu(J X)=i \nu(X)$.

Let $V \in \operatorname{End}(T)$ with $V J+J V=0$;

then, we obtain

$$
V: T^{0,1} \longrightarrow T^{1,0} \quad \mathbb{C}-\text { linear }
$$

simply setting

$$
V(X+i J X)=V(X)-i J V(X)
$$

(i.e., $V$ acts now as $\left.\nu \circ V \circ \bar{\nu}^{-1}\right)$; this identifies canonically $\left(T^{*}\right)^{0,1} \otimes T^{1,0}$ with $\{V \in \operatorname{End}(T) \mid V J+J V=0\}$.

If, moreover, $V=-{ }^{t} V$, then, setting

$$
\alpha(X, Y):=g(V(X), Y),
$$

we obtain $\alpha \in \wedge^{2,0+0,2} T^{*}$ and

$$
\alpha^{2,0}(X, Y)=\frac{1}{2}(\alpha(X, Y)-i \alpha(J X, Y)),
$$

i.e., in terms of the complexified space,

$$
\alpha=\gamma+\bar{\gamma},
$$

with

$$
\gamma \in \wedge^{2,0} T^{*} \quad \gamma(Z, W)=\overline{g(V(\bar{Z}), \bar{W})} .
$$

Let $(M, \kappa, J)$ be a compact Kähler manifold and let $\left(\mathcal{A},[],, \bar{\partial}_{J}\right)$ be the DGLA governing the holomorphic deformation theory of $(M, J)$ :

$$
\mathcal{A}=\bigoplus_{p \in \mathbb{Z}} \mathcal{A}_{p}
$$

where

$$
\mathcal{A}_{p}= \begin{cases}\wedge_{J}^{0, p}(M) \otimes T^{1,0} M, & \text { if } 0 \leq p \leq n \\ 0, & \text { otherwise }\end{cases}
$$

and [, ] is the (complex) Schouten-Nijenhuis bracket (see e.g., [3], p. 152); 
in particular, if $U, V \in \mathcal{A}_{1}$ and, in terms of local holomorphic coordinates $z_{1}, \ldots, z_{n}$

$$
\begin{aligned}
& U=\sum_{j, k=1}^{n} a_{j, \bar{k}} d \bar{z}_{k} \otimes \frac{\partial}{\partial z_{j}}=\sum_{j=1}^{n} a^{(j)} \frac{\partial}{\partial z_{j}} \\
& V=\sum_{j, k=1}^{n} b_{j, \bar{k}} d \bar{z}_{k} \otimes \frac{\partial}{\partial z_{j}}=\sum_{j=1}^{n} b^{(j)} \frac{\partial}{\partial z_{j}}
\end{aligned}
$$

with:

$$
\begin{aligned}
& a^{(j)}=\sum_{k=1}^{n} a_{j \bar{k}} d \bar{z}_{k}, \quad 1 \leq j \leq n \\
& b^{(j)}=\sum_{k=1}^{n} b_{j \bar{k}} d \bar{z}_{k}, \quad 1 \leq j \leq n,
\end{aligned}
$$

then:

$$
\begin{aligned}
{[U, V] } & =\sum_{j, k=1}^{n}\left(a^{(j)} \wedge \frac{\partial b^{(k)}}{\partial z_{j}}+b^{(j)} \wedge \frac{\partial a^{(k)}}{\partial z_{j}}\right) \frac{\partial}{\partial z_{k}} \\
& =\sum_{j, k=1}^{n} \sum_{r<s}\left(a_{j \bar{r}} \frac{\partial b_{k \bar{s}}}{\partial z_{j}}-b_{j \bar{s}} \frac{\partial a_{k \bar{r}}}{\partial z_{j}}\right) d \bar{z}_{r} \wedge d \bar{z}_{s} \otimes \frac{\partial}{\partial z_{k}}
\end{aligned}
$$

and so

$$
[U, V]\left(\frac{\partial}{\partial \bar{z}_{r}}, \frac{\partial}{\partial \bar{z}_{s}}\right)=\left[U\left(\frac{\partial}{\partial \bar{z}_{r}}\right), V\left(\frac{\partial}{\partial \bar{z}_{s}}\right)\right] .
$$

(Of course, for general vector fields $X, Y,[U, V](X, Y) \neq[U(X), V(Y)] !)$. Note that, via $\nu$, we can put the theory in a completely real setting, where: $\mathcal{A}_{p}=\wedge_{J}^{0, p}(M) \otimes T M=\left\{R \in \wedge^{p}(M) \otimes T M \mid R\left(X_{1}, \ldots, J X_{h}, \ldots, X_{p}\right)=\right.$ $\left.-J R\left(X_{1}, \ldots, X_{h}, \ldots, X_{p}\right), \quad 1 \leq h \leq p\right\}$ and, with a slight abuse of notation,

e.g., for $p=0$ :

$$
[R * S]=\nu^{-1}[\nu(R), \nu(S)]
$$

$$
[X * Y]=\frac{1}{2}([X, Y]-[J X, J Y]) .
$$

We shall confine to the complex form of the theory.

Let

and let

$$
\square:=\bar{\partial}_{J} \bar{\partial}_{J}^{*}+\bar{\partial}_{J}^{*} \bar{\partial}_{J}: \mathcal{A} \longrightarrow \mathcal{A}
$$

$$
\begin{aligned}
\nabla^{T M}= & \nabla^{\prime}+\nabla^{\prime \prime}: \operatorname{End}(T M) \longrightarrow \wedge^{1}(M) \otimes \operatorname{End}(T M) \\
& \nabla^{M}: \wedge^{*}(M) \longrightarrow \wedge^{1}(M) \otimes \wedge^{*}(M)
\end{aligned}
$$

be the exterior covariant differential operators with respect to the Levi-Civita connection (which coincides, in the Kähler case, with the Hermitian canonical connection). 
Let $V \in \operatorname{End}(T M)$ such that $J V+V J=0$ and so, in particular

$$
V \in \wedge^{0,1}(M) \otimes T^{1,0} M
$$

let $\alpha=\gamma+\bar{\gamma} \in \wedge^{2,0+0,2}(M, \mathbb{R})$ be defined by:

$$
\alpha(X, Y)=\frac{1}{2} g\left(\left(V-{ }^{t} V\right) X, Y\right)
$$

therefore, in terms of normal local holomorphic coordinates $z_{1}, \ldots, z_{n}$, we have

$$
\begin{gathered}
V=\sum_{j, k=1}^{n} b_{j \bar{k}} d \bar{z}_{k} \otimes \frac{\partial}{\partial z_{j}} \\
{ }^{t}=\sum_{j, k=1}^{n} p_{j \bar{k}} d \bar{z}_{k} \otimes \frac{\partial}{\partial z_{j}}
\end{gathered}
$$

with

$$
p_{j \bar{k}}=\sum_{r, s}^{n} g^{r \bar{j}} b_{s \bar{r}} g_{s \bar{k}}
$$

and

$$
\gamma=\sum_{j<k} c_{j k} d z_{j} \wedge d z_{k}
$$

with

$$
\frac{1}{2}\left(b_{j \bar{k}}-p_{j \bar{k}}\right)=\sum_{r=1}^{n} g^{r \bar{\jmath}} \overline{c_{k r}} .
$$

Therefore, if $B=\left(b_{j \bar{k}}\right), P=\left(p_{j \bar{k}}\right), G=\left(g_{j \bar{k}}\right), C=\left(c_{j k}\right)$, then:

$$
P=\bar{G}^{-1 t} B G
$$

$$
p_{j \bar{k}}=b_{k \bar{j}}+o(|z|)
$$

$$
c_{k j}=\frac{1}{2}\left(\bar{b}_{j \bar{k}}-\bar{b}_{k \bar{j}}\right)+o(|z|)
$$


Note first that, performing the computation at the origin 0 of the system of normal holomorphic coordinates,

$$
\begin{aligned}
\bar{\partial}_{J} V=0 & \Longleftrightarrow \frac{\partial b_{j \bar{k}}}{\partial \bar{z}_{r}}=\frac{\partial b_{j \bar{r}}}{\partial \bar{z}_{k}}, \quad 1 \leq j, k, r \leq n \\
& \Longleftrightarrow \frac{\partial \overline{b_{j \bar{k}}}}{\partial z_{r}}=\frac{\partial \overline{b_{j \bar{r}}}}{\partial z_{k}}, \quad 1 \leq j, k, r \leq n \\
& \Longrightarrow 2 \frac{\partial c_{k j}}{\partial z_{r}}+2 \frac{\partial c_{j r}}{\partial z_{k}}+2 \frac{\partial c_{r k}}{\partial z_{j}}=(\operatorname{by}(4)) \\
& \frac{\partial \overline{b_{j \bar{k}}}}{\partial z_{r}}-\frac{\partial \overline{b_{k \bar{j}}}}{\partial z_{r}}+\frac{\partial \overline{b_{r \bar{j}}}}{\partial z_{k}}-\frac{\partial \overline{b_{j \bar{r}}}}{\partial z_{k}}+\frac{\partial \overline{b_{k \bar{r}}}}{\partial z_{j}}-\frac{\partial \overline{b_{r \bar{k}}}}{\partial z_{j}}=0 \\
& \leq j, k, r \leq n \\
& \Longleftrightarrow \partial_{J} \gamma=0 .
\end{aligned}
$$

We have now the following.

Lemma 3.1. Let $(M, \kappa, J)$ be a compact Kähler manifold; let $\alpha \in \wedge^{2,0+0,2}(M, \mathbb{R}), \alpha=\gamma+\bar{\gamma}=g(V \cdot, \cdot)$ (and so, in particular, $V \in \operatorname{End}(T M)$ with $J V+V J=0$ and $\left.V=-{ }^{t} V\right)$;

then:

(1) $\square V=0 \Longleftrightarrow \nabla^{T M} V=0$

(2) $\nabla^{M} \gamma=0 \Longleftrightarrow \nabla^{T M} V=0$

(3) $\nabla^{T M} V=0 \Longrightarrow[V, V]=0$

Proof. 1: we have

$$
\square V=0 \Longleftrightarrow\left\{\begin{array}{l}
\bar{\partial}_{J} V=0 \\
\bar{\partial}_{J}^{*} V=0 ;
\end{array}\right.
$$

now, in terms of previous notations, and so, once more at 0 of our system of normal local holomorphic coordinates:

$$
b_{j \bar{k}}=\sum_{r=1}^{n} g^{r \bar{\jmath}} \overline{c_{k r}}
$$

with:

$$
\frac{\partial b_{j \bar{k}}}{\partial \bar{z}_{r}}=\frac{\partial b_{j \bar{r}}}{\partial \bar{z}_{k}}, \quad 1 \leq j, k, r \leq n
$$

and the extra condition

$$
b_{k \bar{j}}=-b_{j \bar{k}}+o(|z|)
$$

and so, setting

$$
A_{j k}^{r}:=\frac{\partial b_{j \bar{k}}}{\partial \bar{z}_{r}},
$$


we obtain, by (5) and (6) :

$$
A_{j k}^{r}=-A_{k j}^{r}=A_{j r}^{k}=-A_{k r}^{j}=A_{r k}^{j}=A_{r j}^{k}=-A_{j r}^{k}=-A_{j k}^{r}=0,
$$

i.e.,

$$
\bar{\partial}_{J} V=0 \& V=-{ }^{t} V \Longrightarrow \nabla^{\prime \prime} V=0
$$

also,

$$
\bar{\partial}_{J}^{*} V=0 \Longleftrightarrow \sum_{k=1}^{n} \frac{\partial b_{j \bar{k}}}{\partial z_{k}}=0 \Longleftrightarrow \partial_{J}^{*} \gamma=0, \quad 1 \leq j \leq n ;
$$

consequently,

$$
\square V=0 \Longrightarrow \bar{\square}_{M} \gamma=0 \Longleftrightarrow \Delta \gamma=0
$$

finally,

$$
\bar{\partial} \gamma=0 \Longleftrightarrow \frac{\partial c_{j k}}{\partial \bar{z}_{r}}=0 \Longleftrightarrow \frac{\partial b_{j \bar{k}}}{\partial z_{r}}=0 \Longleftrightarrow \nabla^{\prime} V=0
$$

clearly $\nabla^{T M} V=0 \Longrightarrow \square V=0$ and so all the arrows can be reversed;

2: it's a general Riemannian fact that

$$
\left(\nabla_{X} \alpha\right)(Y, Z)=g\left(\left(\nabla_{X} V\right) Y, Z\right)
$$

3: we have (cf. (1)):

$$
\begin{aligned}
{[V, V]\left(\frac{\partial}{\partial \overline{z_{j}}}, \frac{\partial}{\partial \overline{z_{k}}}\right) } & =\left[V\left(\frac{\partial}{\partial \overline{z_{j}}}\right), V\left(\frac{\partial}{\partial \overline{z_{k}}}\right)\right] \\
& =\sum_{r, s=1}^{n}\left[b_{r \bar{j}} \frac{\partial}{\partial z_{r}}, b_{s \bar{k}} \frac{\partial}{\partial z_{s}}\right] \\
& =\sum_{r, s=1}^{n}\left(b_{r \bar{j}} \frac{\partial b_{s \bar{k}}}{\partial z_{s}}-b_{s \bar{k}} \frac{\partial b_{r \bar{j}}}{\partial z_{s}}\right)=0
\end{aligned}
$$

We have now the following

Lemma 3.2. Let $(M, \kappa, J)$ be a Kähler manifold; given $L \in \wedge^{0,1}(M) \otimes T^{1,0} M$, we have:

$$
\square^{t} L={ }^{t}(\square L)
$$

Proof. Let $z_{1}, \ldots, z_{n}$ be local normal holomorphic coordinates; then at 0 the curvature tensor of $g$ is given by:

$$
R_{a \bar{b} j \bar{k}}=\frac{\partial^{2} g_{a \bar{b}}}{\partial z_{j} \partial \bar{z}_{k}}
$$

and

$$
R_{a \bar{b} j \bar{k}}=R_{j \bar{b} a \bar{k}}=R_{j \bar{k} a \bar{b}}=R_{a \bar{k} j \bar{b}}
$$


moreover, the Ricci tensor is given by

$$
R_{j \bar{k}}=-\sum_{r=1}^{n} R_{j \bar{k} r \bar{r}}
$$

i.e., setting $R=\left(R_{j \bar{k}}\right)$, we have:

$$
R=\frac{1}{2} \Delta_{M} G
$$

Let now

$$
L=\sum_{j, k=1}^{n} l_{j \bar{k}} d \bar{z}_{k} \otimes \frac{\partial}{\partial z_{j}}=\sum_{j=1}^{n} \lambda^{(j)} \otimes \frac{\partial}{\partial z_{j}}
$$

with, clearly, $\lambda^{(j)}=\sum_{k=1}^{n} l_{j \bar{k}} d \bar{z}_{k}, 1 \leq j \leq n$;

then we have (cf. e.g., [3], pp. 101-102):

$$
\bar{\partial}^{*} L=\sum_{j=1}^{n}\left(-* \partial * \lambda^{(j)}-\sum_{r, s=1}^{n} g^{\bar{s} j} *\left(\partial g_{r \bar{s}} \wedge * \lambda^{(r)}\right)\right) \frac{\partial}{\partial z_{j}}
$$

and so, at 0 :

$$
\bar{\partial} \bar{\partial}^{*} L=\sum_{j=1}^{n}\left(-\bar{\partial}(* \partial *) \lambda^{(j)}-\sum_{r=1}^{n} \bar{\partial} *\left(\partial g_{r \bar{\jmath}} \wedge * \lambda^{(r)}\right)\right) \otimes \frac{\partial}{\partial z_{j}}
$$

while

$$
\bar{\partial}^{*} \bar{\partial} L=\sum_{j=1}^{n}\left(-* \partial * \bar{\partial} \lambda^{(j)}\right) \otimes \frac{\partial}{\partial z_{j}}
$$

therefore,

$$
\square L=\sum_{j=1}^{n}\left(\frac{1}{2} \Delta \lambda^{(j)}-\sum_{r=1}^{n} \bar{\partial} *\left(\partial g_{r \overline{\mathrm{j}}} \wedge * \lambda^{(r)}\right)\right) \otimes \frac{\partial}{\partial z_{j}}
$$

now:

$$
\frac{1}{2} \Delta \lambda^{(j)}=\left(\frac{1}{2} \Delta l_{j \bar{k}}-\sum_{r=1}^{n} R_{r \bar{k}} l_{j \bar{r}}\right) d \bar{z}_{k}
$$

and

$$
\bar{\partial} *\left(\partial g_{r \bar{j}} \wedge * \lambda^{(r)}\right)=\sum_{k, p=1}^{n} R_{r \bar{j} p \bar{k}} l_{r \bar{p}} d \bar{z}_{k}
$$


consequently:

$$
\begin{aligned}
\square L= & \sum_{j, k=1}^{n} \frac{1}{2} \Delta_{M} l_{j \bar{k}} d \bar{z}_{k} \otimes \frac{\partial}{\partial z_{j}} \\
& -\sum_{j, k=1}^{n}\left(\sum_{r=1}^{n} R_{r \bar{k}} l_{j \bar{r}}\right) d \bar{z}_{k} \otimes \frac{\partial}{\partial z_{j}} \\
& -\sum_{j, k=1}^{n}\left(\sum_{r, s=1}^{n} R_{r \bar{\jmath} s} l_{r \bar{s}}\right) d \bar{z}_{k} \otimes \frac{\partial}{\partial z_{j}} \\
& \frac{1}{2} \Delta_{M} A-A R-C(A)
\end{aligned}
$$

where $A:=\left(l_{j \bar{k}}\right)$;

now:

$$
{ }^{t} L=\sum_{j, k=1}^{n} p_{j \bar{k}} d \bar{z}_{k} \otimes \frac{\partial}{\partial z_{j}}
$$

with

$$
p_{j \bar{k}}=\sum_{r, s=1}^{n} g^{r \bar{\jmath}} l_{s \bar{r}} g_{s \bar{k}} .
$$

Set $B:=\left(p_{j \bar{k}}\right)$; then:

$$
B=\bar{G}^{-1 t} A G \quad \text { and } \quad B(0)={ }^{t} A(0) ;
$$

now (always at 0$)$ :

$$
\Delta_{M} B=\left(\Delta_{M} \bar{G}^{-1}\right) A+\Delta_{M}{ }^{t} A+A \Delta_{M} G=\Delta_{M}{ }^{t} A+2^{t} A R-2 \bar{R} A ;
$$

consequently:

$$
\begin{aligned}
\square^{t} L & =\frac{1}{2} \Delta_{M}{ }^{t} A+{ }^{t} A R-\bar{R}^{t} A-{ }^{t} A R-C\left({ }^{t} A\right) \\
& =\frac{1}{2} \Delta_{M}{ }^{t} A-\bar{R}^{t} A-C\left({ }^{t} A\right) .
\end{aligned}
$$

Finally, from

- ${ }^{t} R=\bar{R}$,

- $C\left({ }^{t} A\right)=C(A)={ }^{t} C(A)$,

we obtain the result.

Corollary 3.3. If $L \in \wedge^{0,1}(M) \otimes T^{1,0} M$ is $\square$-harmonic, so are $1 / 2\left(L-{ }^{t} L\right)$ and $1 / 2\left(L+{ }^{t} L\right)$.

We are now in position to prove our main result, i.e., Theorem (1.1);

Proof. (a): let $\alpha \in \mathcal{P}^{2,0+0,2}$; write $\alpha=g(V \cdot, \cdot)$; therefore, by Lemma 3.1, $V$ satisfies

- $\nabla^{T M} V=0$ 
- $[V, V]=0$

and clearly this is also the case for $L:=\frac{1}{2} V J$;

in fact:

$$
{ }^{t}(V J)=J V=-V J
$$

and

$$
\nabla^{T M} V J=\left(\nabla^{T M} V\right) J+V\left(\nabla^{T M} J\right)=0:
$$

then apply Lemma 3.1 (3).

Consequently, we have

$$
\bar{\partial}_{J} t L+\frac{1}{2} t^{2}[L, L]=0
$$

and so

$$
J_{t}:=\left(i d_{M}+t L\right) J\left(i d_{M}+t L\right)^{-1}
$$

is a holomorphic structure satisfying $\nabla^{T M} J_{t}=0$;

consequently,

$$
\kappa_{t}:=\frac{1}{2}\left(\kappa+J_{t} \kappa\right)
$$

is parallel, thus it is closed, and $\kappa_{t}=\kappa+t \alpha+o(t)$;

therefore, $\alpha$ is tangent to the curve of Kähler structures $\left(\kappa_{t}, J_{t}\right)$;

$(b)$ : let $\left(\kappa_{t}, J_{t}\right)$ be a curve of Kähler structures with

$$
\kappa_{t}=\kappa+t \alpha+o(t), \quad \alpha \in \wedge^{2,0+0,2}(M) ;
$$

by the basic features of holomorphic deformation theory, we can choose the $\square$-harmonic representative of the class corresponding to the tangent endomorphism to the curve $J_{t}$;

i.e., up to diffeomorphisms, we can assume

$$
J_{t}:=\left(i d_{M}+t L+o(t)\right) J\left(i d_{M}+t L+o(t)\right)^{-1},
$$

with $\square L=0$ and $L J+J L=0$;

it follows from Lemma 3.2 that, if $V=2 J\left(L-{ }^{t} L\right)$, then $\square V=0$ and thus, by Lemma 3.1, $\nabla^{T M} V=0$ and finally, $\nabla^{M} \alpha=0$.

\section{Further Remarks}

As we have already remarked, deformation theory of holomorphic structures ensures that, up to diffeomorphisms, the general (germ of) curve of holomorphic structures on a Hermitian manifold $(M, J, g)$ is of the form

$$
J_{t}:=\left(i d_{M}+t L+o(t)\right) J\left(i d_{M}+t L+o(t)\right)^{-1}
$$

for $L \in \operatorname{End}(T M)$ satisfying $J L+L J=0, \square L=0$;

but, in general, not every such an $L$ gives rise to an actual deformation; in other words, in general, the deformation theory is obstructed.

Let $\mathcal{M}$ be the subset of Ker $\square$ of elements providing actual deformations. 
In the Kähler case, the situation looks somehow neater; in fact:

1) by Corollary 3.3, Ker $\square$ splits as

$$
\operatorname{Ker} \square=\mathfrak{A} \oplus \mathfrak{S}
$$

where, clearly:

$$
\begin{aligned}
& \mathfrak{A}=\left\{L \in \operatorname{Ker} \square \mid L=-{ }^{t} L\right\} \\
& \mathfrak{S}=\left\{L \in \operatorname{Ker} \square \mid L={ }^{t} L\right\}:
\end{aligned}
$$

2) $\mathfrak{A} \subset \mathcal{M}$

and to every $L \in \mathfrak{A}$, we can associate a canonical curve of holomorphic structures:

$$
J_{t}:=\left(i d_{M}+t L\right) J\left(i d_{M}+t L\right)^{-1}
$$

3) note that, in general, for a curve of almost symplectic structures,

$$
\kappa_{t}=\kappa+t \alpha+o(t)
$$

and a curve of $\kappa_{t}$-calibrated complex structures,

$$
J_{t}:=\left(i d_{M}+t L+o(t)\right) J\left(i d_{M}+t L+o(t)\right)^{-1}, \quad(L J+J L=0),
$$

from

$$
\kappa_{t}-J_{t} \kappa_{t}=0
$$

by taking the $t$-derivative at 0 , we obtain

$$
\alpha^{2,0+0,2}=g\left(\left(J L+{ }^{t} L J\right) \cdot, \cdot\right) ;
$$

therefore, if $L \in \mathfrak{S} \cap \mathcal{M}$, and

$$
J_{t}:=\left(i d_{M}+t L+o(t)\right) J\left(i d_{M}+t L+o(t)\right)^{-1}
$$

is a curve of holomorphic structures, then any curve

$$
\kappa_{t}=\kappa+t \alpha+o(t)
$$

of $J_{t}$-Kähler structures satisfies $\alpha \in \wedge_{J}^{1,1}(M)$;

consequently, by Lemma 2.1, it is possible to choose $\kappa_{t}$ of the form

$$
\kappa_{t}=\kappa+o(t)
$$

therefore, the map

$$
\lambda: \mathcal{M} \longrightarrow H^{2}(M, \mathbb{R}) \quad L \mapsto\left[g\left(\left(2 J\left(L-{ }^{t} L\right) \cdot, \cdot\right)\right]\right.
$$

is a linear surjection over $\mathcal{K} / H^{1,1}(M, \mathbb{R})$, which is one-to-one when restricted to $\mathfrak{A}$.

Note that, generically,

$$
\operatorname{Ker} \square=\{0\},
$$

but, within the exceptional range $\operatorname{dim}_{\mathbb{C}} \operatorname{Ker} \square>0$, then, generically,

$$
\mathfrak{A}=\{0\},
$$


i.e., non-trivial $L^{\prime} s \in \wedge^{0,1}(M) \otimes T^{1,0} M$ satisfying $\square L=0$ are generically symmetric: this is a sort of Ayers Rock snow flake principle.

Let us now give a closer look to the case $\mathcal{P}^{2,0+0,2}(M) \neq\{0\}$; first of all recall that, if $(M, \kappa, J)$ is a compact Kähler manifold with Ric $\geq 0$, then any holomorphic form on $M$ is parallel and thus so are harmonic forms in $H^{2,0+0,2}(M, \mathbb{R})$; consequently, for such manifolds

$$
\mathcal{K}=H^{2}(M, \mathbb{R})
$$

(recall also that Ric $>0$ at some point $\Longrightarrow H^{2,0+0,2}(M, \mathbb{R})=0$ ).

Moreover, if on a Kähler manifold $(H, J, \kappa)$ there exists $\alpha \in \wedge^{2.0+0,2}(H, \mathbb{R})$, non-degenerate, satisfying $\nabla^{H} \alpha=0$, then $H$ is hyperkähler, i.e., there exists $K \in \mathfrak{C}_{\kappa}(H)$, satisfying $K J+J K=0$, $\nabla^{H} K=0$ ( $K$ is nothing but the orthogonal factor of the polar decomposition of the endomorphism representing $\alpha$ with respect to the given Kähler metric).

Given $\alpha \in \wedge^{2,0+0,2}(H, \mathbb{R})$, with $\nabla^{M} \alpha=0$, write once more $\alpha=g(V \cdot, \cdot)$, with $V=-{ }^{t} V, J V+V J=0$, and $\nabla^{T M} V=0$; then set:

$$
E(\alpha):=\operatorname{Ker} V, \quad F(\alpha):=(E(\alpha))^{\perp}=\operatorname{Im} V ;
$$

then:

$$
\begin{aligned}
& X \in T M, \quad Y \in E(\alpha) \Longrightarrow \nabla_{X}^{M} Y \in E(\alpha) \\
& X \in T M, \quad Y \in F(\alpha) \Longrightarrow \nabla_{X}^{M} Y \in F(\alpha) .
\end{aligned}
$$

Therefore, the distributions $E(\alpha)$ and $F(\alpha)$ are integrable, $J$-invariant, parallel, and totally geodesic; moreover, if $W \in \operatorname{End}(T M)$ satisfies $\nabla^{T M} W=0$, then

$$
W_{E(\alpha)}:= \begin{cases}W & \text { on } E(\alpha) \\ 0 & \text { on } F(\alpha)\end{cases}
$$

satisfies $\nabla^{T M} W_{E(\alpha)}=0$;

consequently, if $\alpha=h(a)$ has maximal rank for $a \in \mathcal{P}^{2,0+0,2}(M)$, then all elements of $h\left(\mathcal{P}^{2,0+0,2}(M)\right)$ vanish on $E(\alpha)$ and so $E=E(\alpha)$ is unique;

thus, passing to the universal covering $\tilde{M}$, we easily obtain that, from the Kählerian viewpoint

$$
\tilde{M}=N \times H
$$

where $H$ is hyperkähler (and corresponds to $F$ ) and so:

$$
M=N \times \frac{H}{\Gamma}
$$

where $\Gamma$ is a discrete group of holomorphic isometries of $\tilde{M}$; 
summarizing:

$$
\mathcal{P}^{2,0+0,2}(M)\left\{\begin{array}{l}
=\{0\} \Longrightarrow \mathcal{K}=H^{1,1}(M, \mathbb{R}) \\
\neq\{0\} \Longrightarrow M=N \times \frac{H}{\Gamma} .
\end{array}\right.
$$

Note finally that both $\mathfrak{A}$ and $\mathcal{P}^{2,0+0,2}$ are complex vector spaces:

- $\quad \mathcal{J}_{\mathfrak{A}} L:=J L$

- more in general, if $\alpha \in \wedge^{2,0+0,2}(M)$, define

$$
(\mathcal{J} \alpha)(X, Y):=\alpha(J X, Y)=\alpha(X, J Y)
$$

then set, for $a \in \mathcal{P}^{2,0+0,2}$ :

$$
\mathcal{J} a=[\mathcal{J} h(a)]
$$

from $\nabla J=0$, it follows that $\mathcal{P}^{2,0+0,2}$ is a $\mathcal{J}$-complex space; it is clear that $\lambda \circ \mathcal{J}_{\mathfrak{A}}=\mathcal{J} \circ \lambda$.

\section{Examples}

1. Let $M=\mathbb{T}^{2 n}$ be the complex $n$-dimensional torus equipped with the standard Kähler structure $(\kappa, J)$ :

in particular, we have the standard global frame

$$
\left\{\frac{\partial}{\partial x_{1}}, \ldots, \frac{\partial}{\partial x_{n}}, \frac{\partial}{\partial y_{1}}, \ldots, \frac{\partial}{\partial y_{n}}\right\}
$$

with

and standard coframe

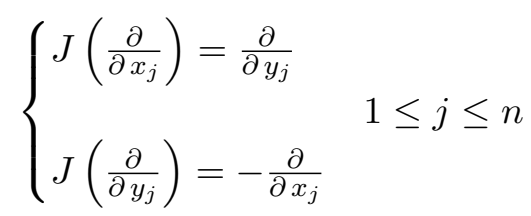

$$
\left\{d x_{1}, \ldots, d x_{n}, d y_{1}, \ldots, d y_{n}\right\}
$$

thus

$$
\kappa=\sum_{h=1}^{n} d y_{h} \wedge d x_{h}
$$

therefore, if $\alpha=d x_{j} \wedge d x_{k}$, then

$$
\alpha=\gamma+\bar{\gamma}+\beta
$$

with

and

$$
\gamma=\frac{1}{4} d z_{j} \wedge d z_{k} \in \wedge^{2,0}\left(\mathbb{T}^{2 n}, \mathbb{C}\right)
$$

$$
\beta=\frac{1}{4}\left(d z_{j} \wedge d \bar{z}_{k}+d \bar{z}_{j} \wedge d z_{k}\right) \in \wedge^{1,1}\left(\mathbb{T}^{2 n}, \mathbb{R}\right) .
$$

Note also that

$$
\alpha^{2,0+0,2}=\frac{1}{2}\left(d x_{j} \wedge d x_{k}-d y_{j} \wedge d y_{k}\right)
$$


(similar formulas for $\left.d y_{j} \wedge d y_{k}, d x_{j} \wedge d y_{k}\right)$;

thus

$$
\gamma=g(V \cdot, \cdot)
$$

for

$$
V=\frac{1}{4}\left(d \bar{z}_{j} \otimes \frac{\partial}{\partial z_{k}}-d \bar{z}_{k} \otimes \frac{\partial}{\partial z_{j}}\right)
$$

or, in real terms,

$$
V=\frac{1}{2}\left(d x_{j} \otimes \frac{\partial}{\partial x_{k}}-d y_{j} \otimes \frac{\partial}{\partial y_{k}}-d x_{k} \otimes \frac{\partial}{\partial x_{j}}+d y_{k} \otimes \frac{\partial}{\partial y_{j}}\right) ;
$$

consider e.g., $n=2, \alpha=4 d x_{1} \wedge d x_{2}$;

then:

$$
V=2\left(\begin{array}{cccc}
0 & -1 & 0 & 0 \\
1 & 0 & 0 & 0 \\
0 & 0 & 0 & 1 \\
0 & 0 & -1 & 0
\end{array}\right)
$$

and so:

$$
L=\frac{1}{2} V J=\left(\begin{array}{cccc}
0 & 0 & 0 & 1 \\
0 & 0 & -1 & 0 \\
0 & 1 & 0 & 0 \\
-1 & 0 & 0 & 0
\end{array}\right)
$$

therefore,

$$
J_{t}=(I+t L) J(I+t L)^{-1}=\frac{1}{1+t^{2}}\left(\begin{array}{cccc}
0 & 2 t & t^{2}-1 & 0 \\
-2 t & 0 & 0 & t^{2}-1 \\
1-t^{2} & 0 & 0 & -2 t \\
0 & 1-t^{2} & 2 t & 0
\end{array}\right)
$$

and

$$
\kappa_{t}=\frac{1}{2}\left(\kappa+J_{t} \kappa\right)=\kappa+t \frac{1-t^{2}}{1+t^{2}} \alpha^{2,0+0,2}+\frac{1}{2} t^{2}\left(t^{2}-6\right) \kappa .
$$

2. First recall that, if $\mathbb{B}:=\{z \in \mathbb{C}|| z \mid<1\}$, then there are no non-trivial parallel $(1,0)$-forms on

$$
\left(\mathbb{B}, \frac{2}{\left(1-|z|^{2}\right)^{2}} d z \wedge d \bar{z}\right)
$$

in fact, given $\gamma=a d z$, we have:

$$
\begin{gathered}
\nabla_{\frac{\partial}{\partial \bar{z}}} \gamma=0 \quad \Longleftrightarrow \quad \frac{\partial a}{\partial \bar{z}}=0 \\
\nabla_{\frac{\partial}{\partial z}} \gamma=0 \quad \Longleftrightarrow \quad \frac{\partial a}{\partial z}-\frac{2 \bar{z}}{1-|z|^{2}} a=0 \quad \Longleftrightarrow \quad a=0 ;
\end{gathered}
$$


consequently, if $\Sigma$ is a Riemann surface covered by $\mathbb{B}$ and equipped with the constant -1 curvature Kähler metric, then there are no non-trivial parallel $(1,0)$-forms on $\Sigma$.

Let now $\Sigma_{g_{k}}, k=1,2$, be compact Riemann surfaces equipped with the constant -1 curvature Kähler metric (and so $g_{k} \geq 2$ ); let $M=\Sigma_{g_{1}} \times \Sigma_{g_{2}}$; then:

$$
H^{1}(M, \Theta)=H^{1}\left(\Sigma_{g_{1}}, \Theta\right) \oplus H^{1}\left(\Sigma_{g_{2}}, \Theta\right)
$$

and, although $H^{2}(M, \Theta) \neq 0$, the holomorphic deformation theory of $M$ is unobstructed and reduces to the deformations of $\Sigma_{g_{1}}$ and $\Sigma_{g_{2}}$ :

- from the previous remarks, it follows quite easily that there are no non-trivial parallel forms in $\wedge^{2,0+0,2}(M, \mathbb{R})$;

therefore, in $M$, we have

$$
\operatorname{Ker} \square=\mathfrak{S} \quad \text { and } \quad \mathcal{K}=H^{1,1}(M, \mathbb{R}) .
$$

\section{References}

[1] P. de Bartolomeis, $\mathbb{Z}_{2}$ and $\mathbb{Z}$-Deformation Theory for Holomorphic and Symplectic Manifolds, Complex, Contact, and Symmetric manifolds, Progress in Mathematics, 234, Birkhäuser, 2005, 75-104.

[2] D. McDuff and D. Salamon, Introduction to Symplectic Topology, Clarendon Press, Oxford, 1995.

[3] J. Morrow and K. Kodaira, Complex Manifolds, Holt, Rinehart and Wiston, Inc., New York, 1971.

Dipartimento di Matematica Applicata G. Sansone

UnIVERsità DI FIRENZE

VIA S. MARTA 3

50139 FIRENZE

ITALY

E-mail address: paolo.debartolomeis@unifi.it

Received Fall 2003, Accepted 10/21/2005. This work was supported by the Project M.I.U.R. "Geometric Properties of Real and Complex Manifolds" and by G.N.S.A.G.A. of I.N.d.A.M. 
